\title{
Ajuste social y escolar de jóvenes víctimas de maltrato infantil en situación de acogimiento residencial
}

\author{
Alexander Muela ${ }^{1 *}$, Nekane Balluerka $^{2}$ y Bárbara Torres ${ }^{2}$ \\ 1 Universidad de Mondragón, España \\ ${ }^{2}$ Universidad del País V asco UPV/EHU, España
}

\begin{abstract}
Resumen: El objetivo del presente trabajo consistió en examinar el ajuste social y escolar de jóvenes que habían sufrido maltrato en la infancia y se encontraban en situación de acogimiento residencial. Además, pretendíamos comprobar si existían diferencias entre los sujetos sometidos a distintas situaciones de desprotección infantil. En el estudio participaron 318 jóvenes residentes en la Comunidad Autónoma del País Vasco de edades comprendidas entre los 13 y los 18 años $(M=15.35, D T=1.68)$ pertenecientes a tres colectivos. El primero de ellos estaba constituido por 69 jóvenes (31 mujeres y 38 varones) víctimas de maltrato infantil que se hallaban bajo una medida de protección de acogimiento residencial. El segundo grupo estaba formado por 75 jóvenes (29 mujeres y 46 varones) víctimas de maltrato infantil que se encontraban en situación de riesgo de desamparo. Por último, el tercer grupo estaba constituido por 174 jóvenes (95 mujeres y 79 varones) que no habían sufrido maltrato. Los resultados pusieron de manifiesto que, independientemente de la situación de desprotección infantil en la que se encontrasen, los jóvenes víctimas de maltrato infantil mostraban mayores dificultades de adaptación social y escolar que los jóvenes que no habían sufrido maltrato en la infancia.

Palabras clave: Acogimiento residencial; adaptación escolar; adaptación social; maltrato infantil; adolescencia.
\end{abstract}

\section{Introducción}

En la Ley Orgánica 1/1996 de Protección Jurídica del Menor, se realiza una distinción entre dos situaciones de desprotección infantil, a saber, las situaciones de riesgo y de desamparo; cada una de ellas da lugar a un grado distinto de intervención por parte de la entidad pública.

Las situaciones de riesgo son aquellas en las que los cuidadores del niño no asumen completamente sus responsabilidades para asegurar el normal desarrollo del niño. Esta situación no conlleva una separación del núcleo familiar, y las actuaciones públicas están orientadas a la eliminación de los factores de riesgo que inciden de forma negativa en el ajuste personal y social de los niños y adolescentes y de sus familias.

En lo que respecta a las situaciones de desamparo, debido a la gravedad de la situación de desprotección, la administración pública competente asume la tutela de la persona menor de edad, adoptando las medidas de protección oportunas. En tanto se mantiene la situación de tutela, la cobertura de las necesidades del niño se garantiza bajo alguna de las siguientes modalidades de guarda: el acogimiento familiar o el acogimiento residencial.

El presente trabajo aborda el ajuste social y escolar de jóvenes que han sufrido maltrato en la infancia y que han sido sometidos a distintas situaciones de desprotección infan-

* Dirección para correspondencia [Correspondence address]: Alexander Muela. Departamento para el Bienestar Infantil. Facultad de Humanidades y Ciencias de la Educación. Universidad de Mondragón. $\mathrm{B}^{\circ}$ Dorleta, s/n, 20540 Eskoriatza (España).

E-mail: amuela@mondragon.edu
Title: Social and school adjustment of adolescent victims of child maltreatment in residential child care.

Abstract: The aim of this study was to examine the social and school adjustment of maltreated adolescents during their childhood who were in residential child care. In addition, it aimed at checking whether there were any differences between those minors who were subjected to different protective measures. The study included 318 adolescents from the Basque Autonomous Community belonging to three groups. The first group was composed by 69 (31 women and 38 men) adolescent victims of serious family maltreatment, who were separated from their biological families, and were in residential child care; the second group consisted of 75 (29 women and 46 men) adolescent victims of moderated maltreatment, living with their biological families under treatment. Finally, the third group was formed by 174 nonmaltreated adolescents ( 95 women and 79 men). The age of the subjects ranged between 13 and 18 years $(M=15.35, S D=$ 1.68). The results showed that, regardless of the child protective measure, those who had been maltreated during their childhood had greater difficulties in social and school adaptation.

Key words: Residential child care; school adjustment; social adjustment; child maltreatment; adolescence.

til. En este sentido, diversos autores destacan que los niños y jóvenes que ingresan en acogimiento residencial, en general, traen consigo una serie de carencias, entre las que se encuentran dificultades de adaptación escolar y de relación social (Martín, Rodríguez y Torbay, 2007). Tales carencias se han puesto de manifiesto en estudios realizados recientemente en nuestro país (Bravo y Del Valle, 2001; Lázaro y López, 2010; Martín, Muñoz de Bustillo, Rodríguez y Pérez, 2008; Moreno, García-Baamonde y Blázquez, 2010).

En lo que se refiere a las investigaciones que han comparado el ajuste escolar de niños en situación de acogimiento residencial con el de niños que residen con su familia biológica, los resultados obtenidos han mostrado que, en comparación con sus compañeros de clase, aquellos que se hallan en acogimiento residencial presentan mayores problemas de atención y de concentración, participan menos en las actividades escolares y tienden a implicarse en actividades alternativas improductivas. Asimismo, presentan mayores problemas sociales, un bajo rendimiento escolar, desobediencia, una conducta agresiva y en el área de juego, raramente interactúan con los niños que no pertenecen al centro residencial (Simsek, Erol, Öztop y Münir, 2007; Vorria, Rutter, Pickles, Wolkind y Hobsbaum, 1998). Además, aquellos que llevan más tiempo en acogimiento residencial muestran relaciones menos armoniosas y de desconfianza con los iguales, y buscan en mayor medida el afecto de los profesores (Vorria et al., 1998).

En lo que respecta a los estudios realizados en la adolescencia, los menores en acogimiento residencial muestran malas relaciones con el profesorado y una conducta agresiva, baja capacidad para resolver conflictos y falta de madurez. 
Además, el profesorado valora negativamente su comportamiento social y su interés por aprender, y les sanciona frecuentemente (Martín et al., 2008). En definitiva, muestran atributos conductuales que interfieren con el correcto desarrollo de las actividades académicas.

Por otra parte, también se ha observado que estos jóvenes son menos elegidos y más rechazados que los demás compañeros para desarrollar actividades académicas, sin embargo, las relaciones con los compañeros parecen ser más adecuadas cuanto más informal es el ámbito en el que se producen, es decir, en actividades de ocio fuera del aula (Bravo y Del Valle, 2003; Martín et al., 2008).

Además, se ha encontrado que los adolescentes acogidos en residencias disponen de una red social más amplia (Bravo y Del Valle, 2003) aunque perciben un menor apoyo social por parte de su red, en especial, por parte de su familia (Lázaro y López, 2010; Bravo y Del Valle, 2003). A su vez, algunos autores afirman que los jóvenes en situación de acogimiento residencial, en comparación con los menores no acogidos, muestran una baja socialización (Carrasco, Rodríguez-Testal y Hesse, 2001; Lázaro y López, 2010), junto a conductas delictivas relacionadas con esa carencia, así como un alto retraimiento social (Carrasco et al., 2001).

Cabe señalar que son pocos los estudios que han utilizado grupos de comparación compuestos por niños o jóvenes bajo distintas medidas de protección a la infancia, y que la mayoría de estos estudios se han realizado con niños. En tales trabajos se constatan mayores problemas escolares en los menores acogidos en residencias que en los sometidos a otras medidas de protección. Por ejemplo, Roy, Rutter y Pickles (2000) compararon la adaptación escolar de niños en acogimiento residencial con la de niños en acogimiento familiar, y con la de niños que no estaban bajo ninguna medida de protección. En los dos grupos que se encontraban bajo una medida de protección se observaron altos índices de hiperactividad y déficit de atención, insociabilidad y problemas emocionales en el ámbito escolar. Sin embargo, cabe señalar que, en comparación con los otros grupos, se hallaron mayores niveles de hiperactividad y déficit de atención en los niños en acogimiento residencial. En dicho trabajo se concluye que el acogimiento residencial predispone a los niños a desarrollar un patrón de hiperactividad y déficit de atención.

Por otra parte, diversos estudios han señalado que estos menores, a pesar de mostrar dificultades, también manifiestan progresos a lo largo del tiempo (Bravo y Del Valle, 2001; 2003; Little, Kohm y Thompson, 2005; Martín et al., 2007, 2008; Martín, Torbay y Rodríguez, 2008). Por ejemplo, se ha encontrado que el trabajo educativo desempeñado en las residencias promueve importantes avances en la autonomía, en el cumplimiento de las obligaciones, en el manejo de los recursos, así como en los cuidados personales y la adaptación al contexto residencial. Sin embargo, en lo que respecta a las relaciones que el niño o el adolescente mantiene con la comunidad (participar en actividades, salir con grupos de amigos, etc.) no se han evidenciado progresos. En lo que se refiere al ámbito escolar, se ha encontrado que gran parte de la población estudiada presenta retraso escolar aunque su predisposición hacia el aprendizaje es adecuada (Bravo y Del Valle, 2001; Martín et al., 2007).

Por último, cabe señalar que las dificultades asociadas al hecho de haber estado sujeto a una medida de protección de acogimiento residencial han sido ampliamente examinadas por investigaciones realizadas en este campo. Según afirman algunos autores (Dixon, 2008; Kendrick, 2005; Stein, 2004), tras su paso por el acogimiento residencial, en muchos de estos jóvenes, se constatan altos niveles de marginación e indigencia, una alta exposición a situaciones de riesgo, gran dependencia de los servicios sociales, pobres resultados académicos, desempleo, dificultades para encontrar un alojamiento estable y una parentalidad precoz. Sin embargo, otros investigadores señalan que la mayoría de los jóvenes en acogimiento residencial no se ven afectados por tales dificultades (Fernández, Álvarez y Bravo, 2003).

En este contexto, el objetivo del presente estudio radica en examinar las habilidades adaptativas y el ajuste personal y escolar de jóvenes que sufrieron maltrato en la infancia, que fueron separados de su familia y que actualmente se encuentran en acogimiento residencial. En lo que se refiere a las habilidades adaptativas, se pretenden examinar sus habilidades sociales para interactuar con sus iguales y con los adultos en el hogar, en la escuela y en la comunidad, así como sus competencias asociadas al logro de metas académicas, sociales o comunitarias. En lo que respecta al ajuste personal, exploraremos el nivel de éxito y el grado de satisfacción que tiene el adolescente acogido en sus relaciones con los demás, la percepción sobre su importancia en la familia, sobre la relación con sus padres y sobre la confianza e interés por parte de los padres. Asimismo, pretendemos analizar la confianza en la propia capacidad para resolver problemas, la seguridad para tomar decisiones y la satisfacción del adolescente consigo mismo, tanto respecto a sus características físicas como a otras más generales. Por último, en cuanto al ajuste escolar, pretendemos conocer la adaptación general del joven al colegio y al proceso educativo. Es decir, el rendimiento académico, la capacidad para el aprendizaje, y la actitud hacia el colegio y los profesores.

Por otra parte, tal y como se ha señalado más arriba, en la actualidad son pocas las investigaciones que han tratado de establecer comparaciones entre jóvenes bajo diferentes medidas de protección a la infancia. Por tal motivo, en esta investigación hemos considerado pertinente establecer una comparación entre las habilidades adaptativas y el ajuste personal y escolar de jóvenes maltratados en situación de acogimiento residencial y de jóvenes que fueron maltratados en la infancia pero no separados de su familia biológica, aunque en la actualidad se encuentren en situación de riesgo de desamparo y sean objeto de una intervención familiar. Por último, hemos considerado oportuno comparar ambos grupos con un grupo de jóvenes que ni fueron objeto de maltrato en la infancia, ni se encuentran en una situación de desprotección infantil. 


\section{Método}

\section{Participantes}

En el estudio participaron 318 jóvenes residentes en la Comunidad Autónoma del País Vasco de edades comprendidas entre los 13 y los 18 años $(M=15.35, D T=1.68)$ pertenecientes a tres colectivos. El primero de ellos estaba constituido por 69 jóvenes (31 mujeres y 38 varones) víctimas de maltrato infantil que se hallaban bajo una medida de protección a la infancia de acogimiento residencial ( $M$ de edad $=15.72, D T=1.64)$. El segundo grupo estaba formado por 75 jóvenes (29 mujeres y 46 varones) víctimas de maltrato infantil que se encontraban en situación de riesgo de desamparo $(M$ de edad $=15.49, D T=1.62)$. Por último, el tercer grupo estaba constituido por 174 jóvenes (95 mujeres y 79 varones) que no se encontraban en situación de desprotección infantil $(M$ de edad $=15.14, D T=1.69)$.

\section{Instrumentos}

Sistema de Identificación y Clasificación del Maltrato infantil (SICMI; Muela, Balluerka y Torres, 2010). El SICMI (Véase la Tabla 1) fue creado para el presente estudio y permite identificar y clasificar el maltrato sufrido por los individuos en diferentes etapas evolutivas. El sistema contempla no sólo los principales tipos de maltrato (Maltrato físico, Negligencia, Abuso sexual y Maltrato emocional), sino también una serie de dimensiones relevantes para la identificación y clasificación de la experiencia maltratante: el tipo de maltrato predominante, la gravedad de la experiencia maltratante, la cronicidad y frecuencia del maltrato, la identidad del maltratador, el estadio del desarrollo en el que ocurre el maltrato y la separación del sujeto de sus figuras de apego. En este trabajo el SICMI se ha utilizado para categorizar el maltrato sufrido en la infancia por los adolescentes participantes en el estudio.

Según el SICMI, una conducta es codificada como maltrato físico cuando el cuidador o adulto responsable inflige daño físico sobre el/la niño/a o adolescente de forma no accidental. Los diferentes tipos de maltrato físico que se conciben son los siguientes: golpear/patear la cara, la cabeza y el cuello; golpear/patear el torso (desde el cuello hasta las piernas excepto las nalgas); golpear/patear las nalgas; golpear/patear miembros, extremidades; el manejo violento del niño (empujar, lanzar, arrastrar); el ahogamiento/la asfixia (con almohada, tapar la boca/nariz, cortar la respiración); las quemaduras/el abrasamiento; y la sacudida.

La negligencia infantil hace referencia a la incapacidad de los cuidadores para cubrir las necesidades físicas básicas de alimentación, vestimenta, higiene, salud (física y mental) y vivienda. Este tipo de maltrato incluye también la incapacidad, por parte de los cuidadores, de proporcionar una adecuada supervisión de la conducta del niño/a o adolescente, la negligencia moral-legal y la negligencia educativa-intelectual.

En el SICMI, una conducta es codificada como abuso sexual cuando se produce "cualquier contacto o intento de contacto sexual entre un cuidador o adulto responsable y un/una niño/a o adolescente, con un objetivo de gratificación sexual o beneficios económicos por parte del adulto" (Barnett, Manly y Cicchetti, 1993, p. 57).

Tabla 1. Criterios para la identificación y clasificación del maltrato infantil incluidos en el SICMI.

\begin{tabular}{|c|c|}
\hline & Clasificación \\
\hline \multirow[t]{4}{*}{ 1. Tipo de maltrato } & Maltrato físico \\
\hline & Negligencia \\
\hline & Abuso Sexual \\
\hline & Maltrato emocional \\
\hline $\begin{array}{l}\text { 2. Tipo de maltrato } \\
\text { predominante }\end{array}$ & $\begin{array}{l}\text { El tipo de maltrato predominante hace re- } \\
\text { ferencia al tipo de maltrato de mayor gra- } \\
\text { vedad, frecuencia y cronicidad. }\end{array}$ \\
\hline \multirow[t]{5}{*}{ 3. Gravedad } & Muy leve \\
\hline & Leve \\
\hline & Moderada \\
\hline & Grave \\
\hline & Muy grave \\
\hline \multirow[t]{3}{*}{ 4. Frecuencia } & Baja \\
\hline & Media \\
\hline & Alta \\
\hline \multirow[t]{3}{*}{ 5. Cronicidad } & Leve \\
\hline & Moderada \\
\hline & Patrón crónico de maltrato \\
\hline \multirow[t]{8}{*}{ 6. Estadio de Desarrollo } & Nacimiento- 6 meses \\
\hline & 7-11 meses \\
\hline & $12-17$ meses (1-1.5 años) \\
\hline & $18-36$ meses (1.5- 3 años) \\
\hline & 37-71 meses (3-5 años) \\
\hline & 72-95 meses (6-7 años) \\
\hline & 96-131 meses (8-10 años) \\
\hline & 132-156 meses (11-13 años) \\
\hline \multirow{7}{*}{$\begin{array}{l}\text { 7.Separación de sus } \\
\text { figuras de apego } \\
\text { 8. Identidad del maltra- } \\
\text { tador }\end{array}$} & Acogimiento familiar \\
\hline & Acogimiento residencial \\
\hline & Madre/Padre biológico \\
\hline & Madrastra/Padrastro/Cuidadores \\
\hline & Otros parientes \\
\hline & Amigo \\
\hline & Persona desconocida/No se sabe \\
\hline
\end{tabular}

En lo que respecta al maltrato emocional, el presente sistema de clasificación, al igual que el Sistema de Clasificación del Maltrato Infantil de Barnett, Manly y Cicchetti (1993), parte del supuesto de que son tres las necesidades emocionales básicas a las que los cuidadores que maltratan a los niños/as o adolescentes no responden adecuadamente, a saber, las necesidades de protección y seguridad psicológica, de aceptación y autoestima, y de autonomía personal adecuada para la edad.

Versión española del Sistema de Evaluación de la Conducta en Niños y Adolescentes (BASC, González, Fernández, Pérez y Santamaría, 2004). El BASC evalúa las habilidades adaptativas y el ajuste personal y escolar. Esta evaluación puede realizarse utilizando distintas fuentes de información: los padres o cuidadores (BASC-P), el personal docente (BASC-T) y el propio sujeto (BASC-S). En lo que se refiere a sus propiedades psicométricas, las tres versiones del instrumento (BASC- 
T, BASC-P y BASC-S) han mostrado una alta consistencia interna (el alpha de Cronbach de las escalas oscila entre .70 y .90) y una adecuada estabilidad temporal (valor promedio de las medianas $=.84 ; .81$ y .76 para el BASC-T, BASC-P y BASC-S, respectivamente) para un intervalo de tiempo de tres meses. En cuanto a su dimensionalidad, el BASC-T y el BASC-S incluyen 18 dimensiones, mientras que el BASC-P integra 15 dimensiones. Asimismo, se han obtenido evidencias de validez de criterio del instrumento (González et al., 2004).

Las habilidades adaptativas hacen referencia a las habilidades prosociales y organizativas, así como a otras de carácter adaptativo del adolescente. Fueron evaluadas a partir de la puntuación obtenida en la dimensión global de Habilidades adaptativas del BASC-P y del BASC-T, que se compone de las escalas Habilidades sociales y Liderazgo. En la escala Habilidades sociales destacan los aspectos interpersonales de la adaptación social. Ejemplos de tales conductas son: admitir errores, felicitar a otros, animar a otros, ofrecer ayuda, iniciar conversaciones adecuadamente y decir "por favor" y "gracias". La escala Liderazgo evalúa una serie de competencias relacionadas con la buena adaptación a la comunidad y a la escuela. Algunos de los elementos de la escala están muy relacionados con las habilidades sociales y otros se refieren a habilidades cognitivas implicadas en la solución de problemas, tales como tener muchas ideas, hacer buenas sugerencias y tomar decisiones fácilmente.

Una puntuación $\mathrm{T}$ igual o inferior a 30 refleja muy bajas habilidades adaptativas, entre $31-40$ se consideran bajas habilidades, entre 41-59 adecuadas habilidades adaptativas, entre 60-69 altas habilidades adaptativas y una puntuación igual o superior a 70 indica unas habilidades adaptativas muy altas.

El ajuste personal se define como el conjunto de estrategias de afrontamiento y de apoyo familiar y social del joven. Fue determinado a partir de la puntuación T obtenida en la dimensión global de Ajuste personal del BASC-S que consta de las escalas Relaciones interpersonales, Relaciones con los padres, Confianza en sí mismo y Autoestima. La escala Relaciones interpersonales mide el nivel de éxito y el grado de satisfacción que tiene el adolescente en las relaciones con los demás, mientras que la escala Relaciones con los padres examina la percepción del individuo sobre su importancia en la familia, la relación con sus padres y la confianza e interés de los padres hacia él. La escala Confianza en sí mismo evalúa la confianza en la propia capacidad para resolver problemas y la seguridad para tomar decisiones. Por último, la escala Autoestima evalúa la satisfacción del adolescente consigo mismo, tanto respecto a sus características físicas como a otras más generales.

Una puntuación $\mathrm{T}$ igual o superior a 70 refleja un ajuste personal muy alto, entre 60-69 se considera que posee un alto ajuste personal, entre 41-59 un adecuado ajuste personal, entre 31-40 un bajo ajuste personal y una puntuación igual o inferior a 30 indica un ajuste personal muy bajo

El desajuste escolar hace referencia a la falta de adaptación del joven en el colegio así como a las dificultades académicas, que incluyen problemas de motivación, atención, aprendizaje y cognición. El nivel de desajuste escolar fue determinado a partir de la puntuación T obtenida en la dimensión global de Problemas escolares del BASC-T, que se compone de las escalas Problemas de atención y Problemas de aprendizaje, y en la Dimensión global Desajuste escolar del BASC-S, que se compone de las escalas Actitud negativa hacia el colegio, Actitud negativa hacia los profesores y Búsqueda de sensaciones. La escala Problemas de atención evalúa la incapacidad para mantener la atención y la tendencia a distraerse fácilmente. La escala Problemas de aprendizaje recoge información de los profesores sobre diversos ámbitos educativos tales como la lectura, la escritura y las matemáticas con el objetivo de detectar dificultades de aprendizaje y de bajo rendimiento. La escala Actitud negativa hacia la escuela refleja la opinión general que tiene el adolescente sobre la utilidad del colegio así como a su nivel de satisfacción en cuestiones relacionadas con el ámbito escolar. La escala Actitud negativa hacia los profesores permite examinar si el sujeto percibe que los profesores son injustos, no le prestan atención, le exigen demasiado o están desmotivados para ayudarle. Por último, la escala Búsqueda de sensaciones evalúa la necesidad de sensaciones y experiencias variadas, nuevas y complejas y el deseo de asumir riesgos físicos y sociales por tales experiencias.

Una puntuación $\mathrm{T}$ igual o superior a 70 refleja un desajuste escolar muy alto, entre 60-69 indica un alto desajuste escolar, entre 41-59 un adecuado ajuste escolar, entre 31-40 alto ajuste escolar y una puntuación igual o inferior a 30 refleja un ajuste escolar muy alto.

\section{Procedimiento}

La investigación se desarrolló en tres fases. La primera fase se centró en la administración de los instrumentos y en la recogida de datos de los jóvenes en situación de acogimiento residencial. En una segunda y tercera fase, se hizo lo propio con los adolescentes que se encontraban en situación de riesgo y con los jóvenes que no estaban en situación de desprotección infantil, respectivamente.

En la primera de ellas, tras obtener el consentimiento tanto por parte de los Servicios de Protección Infantil como por parte de los jóvenes para llevar a cabo el estudio, se tuvo acceso al archivo de expedientes del Departamento de Infancia de las instituciones colaboradoras (Diputación Foral de Guipúzcoa, Diputación Foral de Álava y Ayuntamiento de Vitoria). Una vez confirmados los criterios de inclusión (tener una edad entre 13 y 18 años, haber sufrido maltrato antes de los 13 años y permanecer en situación de acogimiento residencial) y de exclusión (ser un menor extranjero no acompañado o no tener capacidad cognitiva suficiente para poder completar los cuestionarios) que se habían establecido, se llevó a cabo la lectura y el análisis de los expedientes abiertos de los jóvenes seleccionados y se aplicó el SICMI. Tras recoger la información de cada caso, se proce- 
dió a administrar las pruebas de evaluación a los jóvenes, a los educadores de referencia y a los tutores escolares.

La segunda fase se llevó a cabo mediante un procedimiento similar, con la diferencia de que en este caso se contó con la colaboración de los Departamentos de Infancia del Ayuntamiento de Vitoria y San Sebastián y de los padres biológicos. Además, los adolescentes debían cumplir con los criterios de tener una edad entre 13 y 18 años, haber sufrido maltrato antes de los 13 años y estar en situación de riesgo de desamparo. Una vez recogida la información de cada caso, se procedió a administrar a los jóvenes, a uno de sus progenitores y a su tutor escolar las pruebas de evaluación correspondientes.

Por último, en una tercera fase, solicitamos a dos centros escolares públicos su colaboración para disponer de un grupo de jóvenes que no estuvieran en situación de desprotección como criterio de control. La selección de los jóvenes se llevó a cabo a través de un estudio exhaustivo de cada uno de ellos en el que se mantuvieron entrevistas con los orientadores del centro, descartándose cualquier caso sobre el que existiera la más mínima sospecha de haber experimentado maltrato antes de los 13 años. Una vez obtenido el consentimiento de los centros escolares y de los padres de los participantes, y comprobado que los sujetos no habían sufrido maltrato, procedimos a administrar las pruebas de evaluación a los jóvenes, padres y profesores, respectivamente, siguiendo el mismo procedimiento que en los casos anteriores.

\section{Resultados}

Los análisis de datos que se realizaron a fin de responder a los objetivos planteados fueron llevados a cabo con el software SPSS (V.18.0.).

\section{Categorización de la experiencia maltratante a partir del SICMI}

La experiencia maltratante de los jóvenes que se encontraban en situación de acogimiento residencial se caracterizaba por una alta coocurrencia de diferentes tipos de maltrato (88.4\%), siendo el maltrato emocional $(87 \%)$ y la negligencia infantil $(82.6 \%)$ los tipos de mayor incidencia. Si tomamos como referencia el tipo de maltrato predominante, es decir, el maltrato de mayor gravedad, frecuencia y cronicidad, el maltrato emocional $(60.9 \%)$ se reveló como el más frecuente. Cabe señalar que en la mayoría de los casos la experiencia maltratante había sido grave $(88.4 \%)$, no existiendo ningún caso considerado como leve. Además, dicha experiencia se caracterizaba por un patrón crónico de maltrato (79.7\%), una elevada frecuencia de maltrato $(95.7 \%)$, un maltrato ejercido por los padres biológicos $(73.9 \%)$, y una victimización en más de una etapa del desarrollo $(82.6 \%)$.

Con respecto a los adolescentes en situación de riesgo, la mayoría también había experimentado la coocurrencia de diferentes tipos de maltrato $(81.3 \%)$. El maltrato emocional era el de mayor incidencia (84\%), seguido de la negligencia
(77.3\%), el maltrato físico (28\%) y el abuso sexual (5.3\%). El tipo de maltrato predominante fue, una vez más, el maltrato emocional (62.7\%), al que siguieron la negligencia (36\%) y, en menor proporción, el maltrato físico $(1.3 \%)$, no siendo en ningún caso el abuso sexual el tipo de maltrato predominante. A diferencia de los casos que se encontraban en situación de acogimiento residencial, la mayoría de los casos en situación de riesgo habían sido valorados como de gravedad leve o moderada (77.3\%), aunque existía un considerable número de casos valorados como graves (22.7\%). En lo que se refiere a la cronicidad, la mayoría de los casos habían sufrido un patrón crónico de maltrato $(62.7 \%)$. Además, en el 53.3\% de los casos el maltrato había tenido lugar en la etapa infantil. En todos los casos, los padres biológicos habían sido los maltratadores, en el $52 \%$ de los casos el maltrato se había producido en más de una etapa del desarrollo y en un $14.7 \%$ los menores habían sido objeto de separación de los padres biológicos.

\section{Influencia de la situación de desprotección infantil sobre las habilidades adaptativas}

Con el objetivo de examinar si la situación de desprotección ejercía influencia sobre las habilidades adaptativas, se llevó a cabo un análisis multivariante de la varianza, en el que se tomó la situación de desprotección como variable predictora y las habilidades adaptativas observadas por los profesores y por los padres o cuidadores, medidas a través del BASC-T y BASC-P, respectivamente, como variables criterio.

En la Tabla 2 se presentan las puntuaciones medias y las desviaciones típicas en las habilidades adaptativas de los sujetos observadas por los profesores y padres o cuidadores en cada una de las situaciones de desprotección infantil.

Tabla 2. Puntuaciones medias y desviaciones típicas de los sujetos en las habilidades adaptativas observadas por los profesores y padres o cuidadores en cada una de las categorías de desprotección infantil.

\begin{tabular}{|c|c|c|}
\hline $\begin{array}{l}\text { Habilidades } \\
\text { adaptativas }\end{array}$ & $\begin{array}{l}\text { Situación de } \\
\text { desprotección }\end{array}$ & $D T$ \\
\hline $\begin{array}{l}\text { Habilidades adaptativas } \\
\text { observadas por los Padre }\end{array}$ & \multicolumn{2}{|c|}{$\begin{array}{l}\text { No se encuentra en situa-51.639.71 } \\
\text { ción de desprotección }\end{array}$} \\
\hline o cuidadores (Basc P) & $\begin{array}{l}\text { Acogimiento residencial } \\
\text { Situación de riesgo }\end{array}$ & $\begin{array}{ll}38.64 & 10.29 \\
41.14 & 10.85\end{array}$ \\
\hline $\begin{array}{l}\text { Habilidades Adaptativas } \\
\text { observadas por los profe- }\end{array}$ & $\begin{array}{l}\text { No se encuentra en } \\
\text { ción de desprotección }\end{array}$ & -53.44 \\
\hline \multirow[t]{2}{*}{ sores $($ Basc $\mathrm{T})$} & Acogimiento residencial & 45.519 .29 \\
\hline & Situación de riesgo & 44.438 .99 \\
\hline
\end{tabular}

Los resultados pusieron de manifiesto que la situación de desprotección ejercía una influencia estadísticamente significativa sobre las habilidades adaptativas observadas por los padres o cuidadores, $F(2,208)=8.712 ; p=.0001$, aunque no sobre las habilidades adaptativas observadas por los profesores. Tal y como se esperaba, las comparaciones múltiples a posteriori llevadas a cabo mediante la prueba de Tukey mostraron que, en el caso de las habilidades adaptativas observadas por los padres o cuidadores, existían diferencias estadís- 
ticamente significativas entre los adolescentes que no se encontraban en situación de desprotección y los que se encontraban tanto en acogimiento residencial como en situación de riesgo ( $\phi=.0001$ en ambas comparaciones). A su vez, los tamaños del efecto asociados a tales diferencias de medias fueron de gran magnitud tanto en lo que respecta a la primera comparación ( $g$ de Hedges No desprotección-Acogimiento residencial $=1.28$ ), como en lo referente a la segunda ( $g$ de Hedges ${ }_{\text {No }}$ desprotección-Riesgo $=1.04)$. Sin embargo, en contra de lo esperado, no se encontraron diferencias estadísticamente significativas entre los adolescentes que se hallaban en acogimiento residencial y los que se encontraban en situación de riesgo. En este caso, el tamaño del efecto asociado a esa diferencia de medias fue de escasa magnitud ( $g$ de Hedges Acogimiento residencial-Riesgo $=0.25)$.

Por otra parte, aunque no se encontraron diferencias estadísticamente significativas en las habilidades adaptativas observadas por los profesores en función de la situación de desprotección, la reducida potencia de la prueba estadística nos llevó a tomar como referencia un índice alternativo a la prueba de significación, como es el tamaño del efecto (Balluerka, Gómez e Hidalgo, 2005; Balluerka, Vergara y Arnau, 2009). El tamaño del efecto asociada a la diferencia de medias fue de gran magnitud tanto en lo que respecta a la comparación entre los adolescentes que no se encontraban en situación de desprotección y los que se hallaban en acogimiento residencial ( $g$ de Hedges No desprotección-Acogimiento residencial $=0.83)$, como a la comparación entre los adolescentes que no se encontraban en situación de desprotección y los que se hallaban en situación de riesgo ( $g$ de Hedges No desprotección-Riesgo $=0.94)$. Por otra parte, el tamaño del efecto asociado a la diferencia entre los adolescentes que se hallaban en acogimiento residencial y los que estaban en situación de riesgo ( $g$ de Hedges Acogimiento residencial-Riesgo $=0.11$ ) fue pequeño.

\section{Influencia de la situación de desprotección en el ajuste personal del sujeto}

A fin de examinar si la situación de desprotección ejercía influencia sobre el ajuste personal, se llevó a cabo un análisis univariante de la varianza, en el que se tomó la situación de desprotección como variable predictora y el ajuste personal, medido a través del BASC-S, como variable criterio.

En la Tabla 3 se presentan las puntuaciones medias y las desviaciones típicas en el ajuste personal observado por el propio sujeto en cada una de las situaciones de desprotección infantil.

De acuerdo con nuestras expectativas, los resultados mostraron que existían diferencias estadísticamente significativas en el ajuste personal de los sujetos en función de la situación de desprotección a la que habían sido sometidos, $F$ $(2,263)=4.355 ; p=.014$. Tal y como cabía esperar, las comparaciones múltiples entre pares de medias llevadas a cabo mediante la prueba de Tukey revelaron que existían diferencias estadísticamente significativas tanto entre los sujetos que no estaban en una situación de desprotección y los que se encontraban bajo una medida de protección de acogimiento residencial $(p=.0001)$, como entre los sujetos que no estaban en una situación de desprotección y los que se encontraban en situación de riesgo $(p=.0001)$, siendo el ajuste personal en estos dos últimos casos menor que en el primero. Asimismo, no existían diferencias entre los dos grupos de sujetos víctimas de maltrato infantil. De acuerdo con tales resultados, el tamaño del efecto asociado tanto a la primera $(g$ de Hedges No desprotección-Acogimiento residencial $=1.21)$ como a la segunda ( $g$ de Hedges No desprotección-Riesgo $=1.11$ ) comparación de medias fue de gran magnitud, mientras que el asociado a la comparación entre los dos grupos de sujetos que habían sido objeto de maltrato en su infancia fue de escasa magnitud $(g$ de Hedges Acogimiento residencial-Riesgo $=0.10$ ).

Tabla 3. Puntuaciones medias y desviaciones típicas en el ajuste personal percibido por el propio sujeto en cada una de las situaciones de desprotección infantil.

\begin{tabular}{llll}
\hline Ajuste personal & Situación de desprotección & $M$ & $D T$ \\
\hline Ajuste personal & No se encuentra en situación & 52.66 & 7.57 \\
(Basc S) & de desprotección & & \\
& Acogimiento residencial & 41.25 & 12.53 \\
& Situación de riesgo & 42.17 & 11.87 \\
\hline
\end{tabular}

Influencia de la situación de desprotección infantil sobre el desajuste escolar

Con el propósito de examinar si la situación de desprotección ejercía influencia sobre el desajuste escolar, se llevó a cabo un análisis multivariante de la varianza, en el que se tomaron la situación de desprotección como variable predictora y el desajuste escolar observado por el propio sujeto y por los profesores, medido a través del BASC-S y BASC-T, respectivamente, como variables criterio.

En la Tabla 4 se presentan las puntuaciones medias y las desviaciones típicas en el desajuste escolar observado por el propio sujeto y por los profesores en cada una de las situaciones de desprotección infantil.

Tabla 4. Puntuaciones medias y desviaciones típicas en el desajuste escolar percibido por el propio sujeto y por los profesores en cada una de las categorías de desprotección infantil.

Desajuste escolar Situación de desprotección $\quad M \quad D T$

Desajuste escolar No se encuentra en situación de 52.5911 .57 Observado por el desprotección

propio sujeto $\quad$ Acogimiento residencial $\quad 53.2813 .1$

(Basc S) Situación de riesgo $\quad 58.0410 .77$

Desajuste escolar No se encuentra en situación de 46.238 .94

Observado por los desprotección

profesores $\quad$ Acogimiento residencial $\quad 59.9410 .99$ \begin{tabular}{lll} 
(Basc T) & Situación de riesgo & 59.309 .97 \\
\hline
\end{tabular}

De acuerdo a lo esperable, los resultados revelaron que la situación de desprotección ejercía una influencia estadística- 
mente significativa tanto sobre el desajuste escolar observado por el propio sujeto, $F(2,241)=3.282 ; p=.039$, como sobre el observado por los profesores, $F(2,241)=12.081 ; p$ $=.0001$. Las comparaciones múltiples a posteriori llevadas a cabo mediante la prueba de Tukey mostraron que en el caso del desajuste escolar observado por el propio sujeto, existían diferencias estadísticamente significativas entre los adolescentes que no se encontraban en situación de desprotección y los que se encontraban en situación de riesgo $(p=.009)$. El tamaño del efecto asociado a la diferencia de medias fue de magnitud moderada ( $g$ de Hedges ${ }_{\text {No desprotección-Riesgo }}=0.47$ ). Sin embargo, en contra de lo esperado, no se encontraron diferencias estadísticamente significativas entre los adolescentes que no se encontraban en una situación de desprotección y los que se hallaban en acogimiento residencial, ni entre los jóvenes que se hallaban en acogimiento residencial y los que se encontraban en situación de riesgo. De acuerdo con el primer resultado, el tamaño del efecto asociado a la diferencia de medias entre los jóvenes que no se encontraban en situación de desprotección y los que se encontraban en situación de acogimiento residencial fue de escasa magnitud ( $g$ de Hedges No desprotección-Acogimiento residencial $=0.06$ ). No obstante, el tamaño del efecto asociado a la diferencia de medias entre los jóvenes que se encontraban en situación de acogimiento residencial y los que se encontraban en situación de riesgo fue algo mayor $(g$ de Hedges Acogimiento residencial-Riesgo $=0.41)$.

En lo que se refiere al desajuste escolar observado por los profesores, las comparaciones múltiples a posteriori llevadas a cabo mediante la prueba de Tukey mostraron, tal y como cabía esperar, que existían diferencias estadísticamente significativas entre los adolescentes que no se encontraban en situación de desprotección y los que se encontraban tanto en acogimiento residencial como en situación de riesgo ( $p=$ .0001 en ambas comparaciones). Sin embargo, en contra de lo esperado, no existían diferencias estadísticamente significativas entre los dos grupos de sujetos víctimas de maltrato infantil. De acuerdo con tales resultados, el tamaño del efecto asociado tanto a la primera ( $g$ de Hedges No desprotecciónAcogimiento residencial $=1.45)$ como a la segunda ( $g$ de Hedges ${ }_{\text {No }}$ desprotección-Riesgo $=1.38)$ comparación de medias fue de gran magnitud, mientras que el asociado a la comparación entre los dos grupos de sujetos que habían sido objeto de maltrato en su infancia fue de escasa magnitud ( $g$ de Hedges Acogimiento residencial-Riesgo $=0.07$ ).

\section{Discusión}

El objetivo de la presente investigación consistía en examinar las habilidades adaptativas y el ajuste personal y escolar de los jóvenes que habían sufrido maltrato en la infancia, que habían sido separados de su familia biológica y que actualmente se encuentran en acogimiento residencial. Además, pretendíamos analizar si existían diferencias entre los sujetos sometidos a distintas situaciones de desprotección infantil, así como entre éstos y los adolescentes que no han sido víctimas de maltrato en la infancia.

Los resultados pusieron de manifiesto que los jóvenes en situación de acogimiento residencial muestran dificultades de adaptación social y escolar. Además, en comparación con los jóvenes que no han sufrido maltrato, presentan mayores dificultades en dichas áreas. Es decir, muestran menores estrategias de afrontamiento, disponen de un menor apoyo familiar y social, y presentan dificultades académicas que incluyen problemas de motivación, atención, aprendizaje y cognición. También se observaron menores habilidades prosociales y organizativas en este colectivo. Así pues, estos resultados coinciden con los de otras investigaciones recientes que han encontrado problemas similares tanto en niños como en adolescentes (Attar-Schwartz, 2009; Bravo y Del Valle, 2001; Lázaro y López, 2010; Martín et al, 2008; Moreno et al., 2010).

Concretamente, en lo que respecta a las habilidades adaptativas, tanto los educadores de referencia del centro residencial como los profesores consideran que los jóvenes en acogimiento residencial muestran menos habilidades sociales y liderazgo para interactuar satisfactoriamente con los iguales y los adultos en los ámbitos del hogar, de la escuela y de la comunidad. También, al igual que en otras investigaciones (Bravo y Del Valle, 2001; Lázaro y López, 2010), se ha encontrado que estos jóvenes se perciben a sí mismos con un peor ajuste personal caracterizado por un déficit en las estrategias de afrontamiento y de apoyo familiar y social. El joven acogido percibe que tiene menos éxito y muestra un menor grado de satisfacción en las relaciones con los demás, entre las que se encuentran las relaciones con su familia biológica. Además, presenta una menor confianza en sí mismo para resolver problemas y menos seguridad para tomar decisiones, así como una menor autoestima.

Por último, en lo que se refiere al ajuste escolar, de acuerdo con los resultados obtenidos en diversos trabajos llevados a cabo con menores en situación de acogimiento residencial (Bravo y Del Valle, 2001; Martin et al., 2008; Vorria et al., 1998), los profesores consideran que los jóvenes bajo esta medida de protección, en comparación con los que no se encuentran en situación de desprotección, presentan mayores problemas de atención y de aprendizaje. Sin embargo, en la valoración que el propio joven hace sobre su adaptación escolar, no se observan grandes diferencias entre ambos colectivos. A diferencia de lo que observaron Moreno et al. (2010), los adolescentes de nuestro estudio no presentan sentimientos de alienación, hostilidad e insatisfacción respecto del colegio. Tampoco manifiestan sentimientos de resentimiento y antipatía hacia los profesores, ni consideran que éstos son injustos, no les prestan atención o les exigen demasiado. No obstante, sí se ha observado que los jóvenes en acogimiento residencial muestran una mayor tendencia a la búsqueda de sensaciones, es decir, una tendencia a asumir riesgos, lo que puede estar relacionado con problemas de conducta en el colegio. 
Estos resultados nos llevan a concluir que los jóvenes que se encuentran en acogimiento residencial son una población especialmente propensa a presentar un pobre ajuste social y escolar, lo que exige una atención especializada en dichas áreas del desarrollo. Consideramos que los sistemas de protección a la infancia, el sistema escolar, los propios programas individualizados de los centros residenciales y la comunidad en su conjunto deberían garantizar la adecuada integración social y escolar del joven acogido. Algunos autores como Martin et al. (2008) han señalado que es posible que los sistemas de protección a la infancia hayan priorizado la intervención terapéutica frente a los objetivos escolares. Además, hemos de tener en cuenta que actualmente, según datos obtenidos en el Reino Unido, menos de un 6\% de las personas que pasan por algún sistema de protección a la infancia llega a cursar estudios universitarios (Casas y Montserrat, 2009) y que, en gran medida, los jóvenes que pasan por los centros residenciales tienden a presentar dificultades de ajuste psicosocial en la edad adulta (Dixon, 2008; Kendrick, 2005), lo que incrementa la probabilidad de caer en la marginación, la exclusión social y la dependencia de los Servicios Sociales.

En consecuencia, las políticas sociales deberían poner a disposición de estos jóvenes recursos suficientes para prevenir el fracaso escolar y garantizar una educación superior, así como recursos personales de carácter psicosocial para que, una vez finalizada su estancia en las residencias, hayan desarrollado estrategias sociales y personales que les permitan adaptarse a la sociedad. No olvidemos, además, que la mayoría de estos jóvenes, a diferencia de sus iguales, una vez alcanzada la mayoría de edad, han de hacer frente a su emancipación con escasos apoyos familiares y sociales, lo que requiere disponer de habilidades sociales.

Por otra parte, tal y como se ha señalado anteriormente, en el presente estudio se han examinado las diferencias existentes entre el grupo de participantes que había sido objeto de maltrato en la niñez y permanecía en régimen de acogimiento residencial y el grupo que había sido víctima de maltrato infantil y se encontraba en situación de riesgo. Tal comparación se considera de especial relevancia dado que la legislación actual en materia de protección infantil y la distribución de competencias correspondiente en la Comunidad Autónoma del País Vasco entre los servicios de atención a la infancia municipales y forales, distinguen niveles diferentes de intervención en función de la gravedad de los casos. Así, la declaración de una "situación de desamparo" en los casos más graves tiene como consecuencia la asunción de la tutela del menor por parte de la entidad foral competente y la adopción de una medida de protección que implica la separación del niño de su familia biológica (en acogimiento residencial o acogimiento familiar). En cambio, los casos de maltrato evaluados como "leves" o "moderados", son considerados como casos en situación de riesgo de desamparo y la intervención que reciben corre a cargo de los Servicios Sociales municipales, estando orientada a paliar dicho riesgo, de forma que sea posible evitar una separación del menor de su familia actual. En contra de lo esperado, no se han encontrado diferencias entre los dos grupos citados en las habilidades adaptativas, ajuste personal y escolar. De este resultado parece derivarse la necesidad de realizar una profunda reflexión en torno a las estrategias y recursos para la detección y valoración de las situaciones de maltrato infantil, ya que los menores en situación de riesgo muestran el mismo patrón que presenta el grupo de menores maltratados que se encuentran en régimen de acogimiento residencial en habilidades adaptativas, ajuste personal y desajuste escolar, cuando, en teoría, este último colectivo ha sido objeto de una mayor desprotección y privaciones más profundas y tempranas.

Teniendo en cuenta este resultado, cabe pensar que quizá estemos asistiendo a un déficit en la atención a casos de riesgo de desamparo que, si bien en un momento dado son valorados como de gravedad leve o moderada, debido a la situación de desprotección, tienden a cronificarse y pueden sufrir un incremento en la gravedad con el paso del tiempo. Ante esta situación, es posible que desde los Servicios Sociales de Base se estén promoviendo intervenciones que no responden adecuadamente a las consecuencias de la experiencia de desprotección que están experimentando estos menores.

Por otra parte, de los resultados también se puede deducir que la medida de separación y la atención que se procura a los menores en acogimiento residencial no es suficiente para paliar las consecuencias negativas que para el desarrollo supone la experiencia de maltrato infantil y/o la propia separación del menor de su entorno familiar.

Asimismo, es posible concluir que, independientemente de la medida de protección adoptada, ser víctima de malos tratos en la infancia tiene consecuencias muy negativas para el adecuado ajuste psicosocial. Los datos obtenidos coinciden con los resultados de otras investigaciones en las que se ha constatado que los niños que han sufrido maltrato en la infancia muestran importantes problemas en la adolescencia, tales como consumo de drogas y alcohol, fracaso escolar, dificultades en la socialización, diversos problemas de conducta y un mayor riesgo de desarrollar trastornos de la personalidad (Evans, Hawton y Rodham, 2005; Muela, 2008; Shaffer, Huston y Egeland, 2008; Turner, Finkelhor y Ormrod, 2006). Además, de acuerdo con nuestros resultados, distintos estudios han puesto de manifiesto que los adolescentes maltratados, en comparación con los no maltratados, presentan mayores dificultades en las variables citadas (Lansford et al. 2002; Malinosky-Rummell y Hansen, 1993).

Estos datos avalan el argumento de varios autores (Attar-Schwartz, 2009; Casas y Montserrat, 2009) que afirman que los jóvenes que están o han estado en algún sistema de protección a la infancia se encuentran en riesgo de exclusión social, situación que, tal y como afirman Casas y Montserrat (2009), reúne, en España, todas las características de un problema social no legitimado. En línea con este argumento, se podría decir que los sistemas educativos, independientemente de la medida de protección adoptada, no reconocen aún a los jóvenes víctimas de maltrato como un conjunto de la po- 
blación escolar con necesidades especiales. Por lo tanto, es necesario activar programas especializados con la población maltratada para garantizar una mejor adaptación escolar. Por otra parte, unido a esta necesidad, consideramos también oportuno potenciar intervenciones dirigidas a mejorar las capacidades de ajuste social de estos jóvenes. El desarrollo de sus capacidades resulta clave para incrementar la probabilidad de éxito social y profesional, es decir, un ajuste personal más satisfactorio y más adaptado a su comunidad. En definitiva, en un sentido amplio, pensamos que el bienestar integral de los menores que son objeto de la atención de los Servicios de Protección Infantil sigue siendo una asignatura pendiente (Sullivan y van Zyl, 2008).

No queremos finalizar el trabajo sin señalar algunas de sus limitaciones. En primer lugar, hemos de decir que los resultados aquí obtenidos tendrían mayor validez externa si se replicaran con muestras más amplias de sujetos. No obstante, resulta muy complicado llevar a cabo un estudio de estas características con jóvenes víctimas de maltrato que se encuentran bajo diferentes medidas protectoras. El acceso a estos participantes se plantea cada vez más difícil puesto que el nivel de participación suele ser escaso y conseguir la colaboración de las instituciones no está exento de dificultades. Además, si ya es difícil obtener la cooperación de los participantes, debido a aspectos legales relacionados con la Ley de Protección de Datos, y a pesar del escrupuloso tratamiento de estos datos por parte de los investigadores, es muy complicado tener acceso a los expedientes de los menores en si-

\section{Referencias}

Attar-Schwartz, S. (2009). School functioning of children in residential care: The contributions of multilevel correlates. Child Abuse and Neglect, 33(7), 429-440.

Balluerka, N., Gómez, J. e Hidalgo, M. D. (2005). The controversy over null hypothesis significance testing revisited. Methodology. European Journal of Research Methods for the Behavioral and Social Sciences, 1 (2), 55-70.

Balluerka, N., Vergara, A. y Arnau, J. (2009). Calculating the main alternatives to null-hypothesis-significance testing in between-subject experimental designs. Psicothema, 21 (1), 141-151.

Barnett, D., Manly, J. T. y Cicchetti, D. (1993). Defining child maltreatment: The interface between policy and research. In D. Cicchetti y S. L. Toth (Eds.), Child abuse, child development, and social policy (pp. 7-74). Norwood, NJ: Ablex.

Bravo, A. y Del Valle, J. F. (2001). Evaluación de la integración social en acogimiento residencial. Psicothema, 13(2), 197-204.

Bravo, A. y Del Valle, J. F. (2003). Las redes de apoyo social de los adolescentes acogidos en residencias de protección. Un análisis comparativo con población normativa. Psicothema, 15(1), 136-142.

Carrasco, M. A., Rodríguez-Testal, J. F. y Hesse, B. (2001). Problemas de conducta de una muestra de menores institucionalizados con antecedentes de maltrato. Child Abuse and Neglect, 25(6), 819-838.

Casas, F. y Montserrat, C. (2009). Sistema educativo e igualdad de oportunidades entre los jóvenes tutelados: estudios recientes en el Reino Unido. Psicothema, 21(4), 543-547.

Dixon, J. (2008). Young people leaving residential care: Experiences and outcomes. In A. Kendrick (Ed.), Residential child care: prospects and challenges (pp. 76-89). London: Jessica Kingsley Publishers.

Evans, E., Hawton, K. y Rodham, K. (2005). Suicidal phenomena and abuse in adolescents: A review of epidemiological studies. Child Abuse and $\mathrm{Ne}$ glect, 29(1), 45-58. tuación de desprotección. Si a esto le añadimos la necesaria colaboración de los educadores, el personal docente y los padres de los menores, el estudio queda aún mucho más limitado pues existe un gran riesgo de mortandad experimental, tal y como ha sucedido en nuestro estudio.

En segundo lugar, sería interesante abordar este objeto de estudio mediante un diseño longitudinal que permitiese el seguimiento de los mismos sujetos víctimas de maltrato desde la infancia, pasando por la edad escolar, la adolescencia temprana y la adolescencia tardía. Esto posibilitaría obtener una información precisa acerca de la evolución en las habilidades adaptativas, el ajuste personal y el desajuste escolar. Además, en caso de que el menor fuera separado de su contexto familiar, se podría obtener información antes de la separación, después de la misma y durante el tiempo en el que se encontrase bajo una medida de protección separado de su familia biológica, o incluso más allá, si se diera el caso de retorno con su familia de origen.

Por último, consideramos que para profundizar en el estudio de las habilidades adaptativas y el ajuste personal y escolar en jóvenes víctimas de maltrato infantil, además de las muestras estudiadas, deberían examinarse otros colectivos que cumplan el criterio de haber sido víctimas de maltrato infantil antes de los 13 años, como, por ejemplo, adolescentes víctimas de maltrato infantil en régimen de acogimiento familiar, menores extranjeros no acompañados en situación de acogimiento residencial y jóvenes infractores con medida protectora.

Fernández, J., Álvarez, E. y Bravo, A. (2003). Evaluación de resultados a largo plazo en acogimiento residencial de protección a la infancia. Infancia y Aprendizaje, 26(2), 235-249.

González, J. Fernández, S., Pérez E. y Santamaría P. (2004). BASC, sistema de evaluación de la conducta de niños y adolescentes. Madrid: TEA Ediciones.

Kendrick, A. (2005). Social exclusion and social inclusion: Themes and issues in residential child care. In D. Crimmens y I. Milligan (Eds.), Facing forward. Residential child care in the 21st. century (pp. 7-18). Dorset: Russel House Publishing.

Lansford, J. E., Dodge, K. A., Pettit, G. S., Bates, J. E., Crozier, J. y Kaplow, J. (2002). A 12-year prospective study of the long-term effects of early child physical maltreatment on psychological, behavioral, and academic problems in adolescence. Archives of Pediatrics and Adolescent Medicine, 156, 824-830.

Lázaro, S. y López, F. (2008). Continuidad de los efectos del maltrato durante la infancia en adolescentes acogidos en centros de protección. Infancia y Aprendizaje, 33(2), 255-268.

Little, M., Kohm, A. y Thompson, R. (2005). The impact of residential placement on child development: research and policy implications International Journal of Social Welfare, 14, 200-209.

Malinosky-Rummell, R. y Hansen, D. J. (1993). Long-term consequences of childhood physical abuse. Psychological Bulletin, 114(1), 68-79.

Martín, E. y Dávila, L. M. (2008). Redes de apoyo social y adaptación de los menores en acogimiento residencial. Psicothema, 20(2), 229-235.

Martín, E., Muñoz de Bustillo, M. C., Rodríguez, T. y Pérez, Y. (2008). De la residencia a la escuela: la integración social de los menores en acogimiento residencial con el grupo de iguales en el contexto escolar. Psicotbema, 20(3), 376-382.

Martín, E., Rodríguez, T. y Torbay, A. (2007). Evaluación diferencial de los programas de acogimiento residencial para menores. Psicothema, 19(3), 406-412. 
Martín, E., Torbay, A. y Rodríguez, T. (2008). Cooperación familiar y vinculación del menor con la familia en los programas de acogimiento residencial. Anales de Psicología, 24(1), 25-32.

Moreno, J. M., García-Baamonde, Mª . E. y Blázquez, M. (2010). Desarrollo y adaptación escolar en niños en acogimiento residencial. Anales de Psicologia, 26(1), 189-196.

Muela, A. (2008). Hacia un sistema de clasificación nosológico de maltrato infantil. Anales de Psicología, 24(1), 77-87.

Muela, A., Balluerka, N. y Torres, B. (2010). Sistema de identificación y clasificación del maltrato infantil. San Sebastián: Orekagune gizartean eta komunitatean eskuhartzeko elkartea.

Roy, P., Rutter, M. y Pickles, A. (2000). Institutional care: risk from family background or pattern of rearing? Journal of Child Psychology and Psychiatry, 41(139-149).

Roy, P., Rutter, M. y Pickles, A. (2004). Institutional care: associations between overactivity and lack of selectivity in social relationships. Journal of Child Psychology and Psychiatry, 45(4), 866-873.

Shaffer, A., Huston, L. y Egeland, B. (2008). Identification for child maltreatment using prospective and self-report methodologies: A compari- son of maltreatment incidence and relation to later psychopathology. Child Abuse and Neglect, 32, 682-692.

Simsek, Z., Erol, N., Öztop, D. y Münir, K. (2007). Prevalence and Predictors of Behavioral Problems Reported by Teachers: Turkish Orphans Study. Children and Youth Services Reviews, 29, 883-899.

Stein, M. (2004). What Works for Young People Leaving Care? Barnardos: Ilford. Sullivan, D. J. y van Zyl, M. A. (2008). The well-being of children in foster care: exploring physical and mental health needs. Children and Youth Services Review, 30, 774-786.

Turner, H. A., Finkelhor, D. y Ormrod, R. (2006). The effect of lifetime victimization on the mental health of children and adolescents. Social Science and Medicine, 62, 13-27.

Vorria, P., Rutter, M., Pickles, A., Wolkind, S. y Hobsbaum, A. (1998). A comparative study of Greek children in long-term residential group care and in two-parent families: I. Social, emotional, and behavioural differences. Journal of Child Psychology and Psychiatry, 39, 225-245.

(Articulo recibido: 7-4-2011, revisado: 31-08-2011, aceptado: 19-02-2012) 